УДК 336.64; JEL D14, L 86, L 96

DOI: $10.17223 / 22229388 / 25 / 1$

Д.А. Ендовицкий, И.В. Фролов, Р.Р. Рахматулина

СРАВНИТЕЛЬНЫЙ АНАЛИЗ ПОДХОДОВ К КОЛИЧЕСТВЕННОЙ ОЦЕНКЕ

\section{КРЕДИТОСПОСОБНОСТИ ЗАЕМЩИКА}

Проведен обзор существующих в современной экономической литературе и практике подходов к количественной оценке кредитоспособности заемщика. Выделены критерии количественной оценки интерпретации уровня кредитоспособности, выявлены достоинства и недостатки современных кредитных рейтингов. Предложен универсальный количественный показатель оценки кредитоспособности заемщика, релевантный ее экономи ческому содержанию.

Ключевые слова: кредитоспособность заемщика, кредитный риск, кредитный рейтинг, показатель, модель.

Большинство современных методик анализа кредитоспособности предполагают ее количественную оценку как вероятности или условного показателя (класса, группы), который может быть интерпретирован как вероятность. Соответственно кредитный риск и кредитоспособность можно в первом приближении рассматривать как две взаимодополняющие вероятности.

Исследуя проблему количественной интерпретации понятия кредитоспособности, мы сформулировали минимальный набор требований, которым должны отвечать как сама интерпретация, так и оценки, полученные на ее основе:

- экономическое содержание. Количественная оценка должна не только соотноситься с уровнем кредитоспособности, но и отражать реально наблюдаемое и измеримое явление, в противном случае она будет неверифицируемой. Одно из практических следствий данного требования состоит в том, что показатель должен по возможности выражаться в конкретных, а не в условных единицах измерения;

- комплексность. Оценка должна отражать уровень кредитоспособности в целом, а не один из факторов кредитоспособности. Для вынесения суждения об уровне кредитоспособности должно быть достаточно одного количественного показателя, в противном случае он не может претендовать на роль оценки кредитоспособности. Отказ от требования комплексности недопустим, пока не доказана невозможность получения такой оценки. Обоснованное доказательство невозможности комплексной оценки ставит под сомнение рассматриваемое понятие как самостоятельное и реально существующее явление или характеристику;

- релевантность определению. Если в определении понятия не указаны определенные измеримые характеристики либо они указаны только в качестве факторов, оценка должна однозначно опознаваться аналитиками как уровень кредитоспособности. Если в определении уже дана однозначная количественная характеристика, задача построения количественной оценки не возникает. Выполнение предыдущего требования комплексности тесно связано с релевантностью, но не является ее достаточным условием;

- использование для качественно иных задач. Оценка должна быть потенциально полезной для решения других задач экономического анализа в качестве исходного показателя (фактора). Показатель, пригодный для решения единственной задачи, можно рассматривать как неотъемлемый промежуточный этап решения, но не как самостоятельный элемент единой системы показателей. Хотя круг задач, где могла бы использоваться оценка, изначально определить затруднительно, ее использование не должно быть затруднено излишними преобразованиями или отсутствием надежных зависимостей. Выполнение требования экономического содержания связано с полезностью оценки для иных задач и является ее необходимым условием;

- использование для принятия решений. Показатель должен прямо и непосредственно использоваться в принятии решений, предполагающих оценку кредитоспособности, т.е. как минимум в решении о выдаче кредита. Данное требование значимо именно для оценки кредитоспособности и вытекает из ее определения. Выполнение требования комплексности тесно связано с полезностью показателя для принятия решений и является ее необходимым условием. Следует учитывать, что адаптация показателя под конкретную задачу может войти в противоречие с полезностью для качественно иных задач. Например, показатель кредитоспособности мог бы выглядеть как бинарный критерий («да / нет»), но в таком виде он будет непригоден для большинства иных целей.

Приведенный выше перечень требований не рассматривается как универсальный и закрытый. Он выведен, главным образом, из соображений полезности будущей оценки. Далее мы рассмотрим наиболее распространенные из существующих подходов к оценке кредитоспособности с позиции сформулированных требований, начав с обзора возможностей показателя, прямо вытекающего из содержания определения - вероятности дефолта заемщика. Здесь и далее мы используем термин «дефолт» в значении, наиболее распространенном в зарубежной экономической литературе по исследуемому вопросу: вероят- 
ность прекращения или неприемлемой задержки выплат по обязательствам. Следует также отметить, что понятие «кредитоспособность» используется в отечественной литературе; зарубежные исследователи оперируют терминами «дефолт» (default), «прекращение платежей» (failure) и «кредитный риск».

Значение численной интерпретации уровня кредитоспособности заключается в возможности перехода от качественного определения к количественным показателям, что создает основу для применения методов экономического анализа. Однако с учетом задач анализа интерпретации кредитоспособности как вероятности добросовестного исполнения обязательств по кредитному договору может быть недостаточно. В частности, для того чтобы перейти к определению размера резерва под возможные потери по кредиту, необходимо располагать также данными об объемах кредитования и о графике распределения ожидаемых потерь при недобросовестном исполнении обязательств.

В соответствии с п. 5.5.17-5.5.20 МСФО (IFRS) 9 «Финансовые инструменты» [9] резерв под обесценение финансовых активов, таких как выданные кредиты, рассчитывается как сумма дисконтированных по первоначальной эффективной процентной ставке отклонений от предусмотренных договором денежных потоков.

Кредитный убыток возникает даже тогда, когда банк ожидает получить всю сумму, но позже установленных сроков. Ожидаемые к получению денежные потоки рекомендовано определять при помощи интервальных оценок и взвешивать по вероятности.

Представим пример вероятностного распределения отдельного денежного поступления на графике (рис. 1).

При добросовестном исполнении обязательств с вероятностью $P=1$ поступления $R$ не превысят суммы по договору $D_{i}$ (при недобросо- вестном исполнении поступления могут увеличиться на сумму штрафных санкций); кроме того, поступления не могут быть отрицательными $(R \geq 0)$. Обычно также существует отличная от нуля вероятность, что заемщик полностью выполнит свои обязательства, что отражает горизонтальный участок кривой вблизи точки $D_{i}$, и часто допускается некоторая вероятность, что поступления будут нулевыми (горизонтальный участок кривой вблизи нуля). Если вероятность полного отсутствия поступлений принимается нулевой, кривая будет начинаться выше, в точке, соответствующей вероятности минимального из ожидаемых поступлений. На промежуточном интервале $\left(0 ; D_{i}\right)$ кривая монотонно поднимается от минимального значения до 1 , при этом она может иметь несколько точек перегиба или изломы, характерные для интервальной оценки вероятностей.

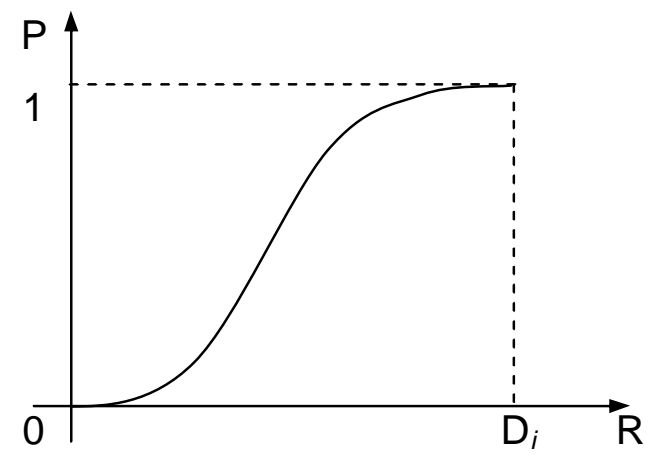

Рис. 1. Распределение вероятности поступлений от заемщика по отдельному платежу

Для расчета кредитного убытка по данному платежу в соответствии с требованиями МСФО (IAS) 39 и МСФО (IFRS) 9 необходимо перемножить ожидаемые к поступлению суммы на вероятности их поступления. Упрощенный пример такого расчета для трех ожидаемых поступлений представлен в табл. 1 .

Таблица 1. Распределение вероятностей денежных поступлений для расчета кредитных убытков

\begin{tabular}{|c|c|c|c|c|c|c|c|c|c|}
\hline \multirow{3}{*}{ Период } & \multicolumn{9}{|c|}{ Денежные потоки по периодам } \\
\hline & \multicolumn{3}{|c|}{1} & \multicolumn{3}{|c|}{2} & \multicolumn{3}{|c|}{3} \\
\hline & $\mathrm{P}$ & $\mathrm{FV}$ & $\mathrm{PV}$ & $\mathrm{P}$ & FV & $\mathrm{PV}$ & $\mathrm{P}$ & $\mathrm{FV}$ & $\mathrm{PV}$ \\
\hline \multicolumn{10}{|l|}{0} \\
\hline 1 & 0,99 & 1360 & 1214 & & & & & & \\
\hline 2 & 0,01 & 1360 & 1084 & 0,97 & 1240 & 989 & & & \\
\hline 3 & & & & 0,01 & 1240 & 883 & 0,95 & 1120 & 797 \\
\hline 4 & & & & 0,01 & 1240 & 788 & 0,03 & 1120 & 712 \\
\hline$\infty$ & & & & 0,01 & 1240 & 0 & 0,02 & 1120 & 0 \\
\hline Взвешенг & & \multicolumn{2}{|c|}{1213} & \multicolumn{3}{|c|}{976} & \multicolumn{3}{|c|}{779} \\
\hline
\end{tabular}

Примечания:

$\mathrm{P}$ - вероятность поступления данной суммы в указанный период, коэф.;

FV - фактическая сумма поступления, ожидаемого в данный период, д.е.;

PV - дисконтированная сумма ожидаемого поступления, д.е.

В данном примере заемщику выдается кредит в сумме 3000 д.е. сроком на 3 периода при эффективной процентной ставке $12 \%$. С вероятностью $99 \%$ первый платеж по кредиту в сумме 1360 д.е. (1/3 основной суммы долга + проценты) поступит в срок. C вероятностью $1 \%$ допускается, что платеж поступит с запозданием, но в полном объеме и не позднее конца следующего периода. Поэтому взвешенная по вероятностям дисконтированная сумма ожидаемых поступлений в счет первого периода составит: 
$0,99 \times 1360 /(1+0,12)^{1}+0,01 \times 1360 /$

$(1+0,12)^{2}=1212,98$ д.е.

Проведя аналогичные расчеты за 2-й и 3-й периоды, получим общую сумму взвешенных дисконтированных ожидаемых поступлений 2967,24 д.е., что соответствует кредитному убытку (3000 $2967,24)=32,76$ д.е. $(1,09 \%$ от суммы кредита $)$.

Оценка вероятности нарушения кредитором обязательств требует дополнительных данных об условных вероятностях событий. На практике нарушение обязательств в каком-либо периоде увеличивает вероятность нарушений в последующих периодах, и наоборот. Поэтому упрощающее допущение о независимости нарушений даст достаточно осторожную оценку вероятности соблюдения заемщиком всех обязательств:

$$
P=0,99 \times 0,97 \times 0,95=0,9123 \text { или } 91,23 \%,
$$

откуда оценка риска нарушения договора составит 8,77 \%. Чтобы в дальнейшем не работать с полным набором значений табл. 1, удобно сопоставить рассчитанной вероятности 8,77 \% некоторую среднюю сумму потерь, или нормированный ожидаемый убыток (HOУ):

Ожидаемые поступления при нарушении договора $=(2967,24-3000 \times 0,9123) /(1-0,9123)=$ $=2626,56$ д.е., $Н О У=3000-2626,56=373,44$ д.е.

Чтобы перейти от нормированного ожидаемого убытка к среднему ожидаемому кредитному убытку, под который формируется резерв, достаточно умножить $Н О У$ на вероятность нарушения договора:

$$
0 \times 0,9123+373,44 \times 0,0877 \approx 32,76 \text { д.е. }
$$

Таким образом, хотя значение вероятности нарушения условий кредитного договора само по себе и недостаточно для расчета резерва под возможные потери, для решения указанной задачи достаточно дополнить его единственным показателем - суммой нормированного ожидаемого убытка. Данный подход соответствует требованиям МСФО (IFRS) 9, согласно которым следует рассматривать минимум два сценария: возникновение и отсутствие кредитного убытка [9. П. 5.5.18].

Удобство использования НОУ при расчете резерва дает ему значимые преимущества в сравнении с другими стоимостными показателями риска, например, VAR (value-at-risk). Показатель $V A R$ отражает наибольший убыток, ожидаемый с заданной вероятностью, т.е. на нижней границе доверительного интервала [16. Р. 51]. Обычно рассматривается VAR для $95 \%$-го или $99 \%$-го доверительного интервала. Хотя такой подход и позволяет сократить количество показателей с двух (вероятность и убыток) до одного, значение $V A R$ отражает конкретный «умеренно пессимистический» сценарий и способно ввести в заблуждение (средний убыток значительно меньше, чем $V A R)$. К тому же для таких финансовых инструментов, как кредиты, практически невозможно обоснованно построить доверительный интервал (в отличие от облигаций и производных финансовых инструментов на организованных торгах).

Необходимо уточнить, что в Российской Федерации кредитные и некредитные финансовые организации формируют резервы в соответствии с требованиями нормативных правовых актов Банка России, которые могут отличаться от положений Международных стандартов финансовой отчетности, дополнять либо конкретизировать их. В частности, кредитными организациями резервы по ссудной задолженности формируются в соответствии с Положением, утвержденным Банком России 26.03.2004 г. № 254-п [2] (далее - Положение № 2 54-п). Данное Положение предписывает определять размер резерва (в общем случае, при отсутствии иных существенных обстоятельств) на основе двух факторов: финансового положения заемщика и качества обслуживания долга. Каждый из факторов оценивается по трехуровневой шкале (табл. 2).

Таблица 2. Определение размера резерва в соответствии с Положением № 254-п, п. 3.9
\begin{tabular}{|c|c|c|c|}
\hline $\begin{array}{c}\text { Финансовое по- } \\
\text { ложение заемщи- } \\
\text { ка }\end{array}$ & \multicolumn{3}{|c|}{ Качество обслуживания долга, \% } \\
\cline { 2 - 4 } & хорошее & среднее & неудовлетворительное \\
\hline Хорошее & 0 & $1 \ldots 20$ & $21 \ldots 50$ \\
\hline Среднее & $1 \ldots 20$ & $21 \ldots 50$ & $51 \ldots 100$ \\
\hline Плохое & $21 \ldots 50$ & $51 \ldots 100$ & 100 \\
\hline
\end{tabular}

С одной стороны, такой подход можно рассматривать как удобную эмпирическую процедуру, позволяющую избежать прогнозирования ожидаемых денежных потоков по каждой выданной ссуде. Кроме того, такую оценку требуется осуществлять индивидуально только в отношении существенных статей, незначительные ссуды (составляющие в совокупности по одному заемщику не более 0,5 \% от собственного капитала кредитной организации [2. П. 5.1]) допускается группировать в портфели однородных ссуд.
С другой стороны, требование Положения № 254-П о создании резерва от основной суммы долга, без процентов [2. П. 3.11], входит в противоречие с MСФО (IFRS) 9, согласно которому кредитные убытки рассчитываются по всем поступлениям с учетом временной стоимости денег, что может привести к необходимости корректировок при составлении финансовой отчетности. Кроме того, градация размеров резервов по II-IV категориям качества ссуд (пороговые значения 20 
и 50\%) не представляется обоснованной даже эмпирически и может входить в противоречие с реальным размером кредитных убытков. Негативное влияние данных ограничений компенсируется разрешением определять конкретный размер резерва внутри диапазона, а также понижать или повышать в отдельных случаях категорию качества ссуды на один уровень, так что в конкретных ситуациях можно попытаться согласовать требования национальных и международных стандартов.

С 01.10.2015 г. вступило в силу Положение о порядке расчета величины кредитного риска на основе внутренних рейтингов, утвержденное Банком России 06.08.2015 г. № 483-п (далее - Положение № 483-п), реализующее подход к формированию резервов на основе современных статистических методов. Переход к применению данного порядка допускается только для банков и только по индивидуальному разрешению Банка России. Для получения разрешения банк обязан, в частности, располагать внутренней рейтинговой системой, действующей не менее 3 лет, в том числе не менее 2 лет - в отношении $50 \%$ и более от суммы кредитных требований [1. П. 1.7, 1.8, 1.12]. В целях подготовки к переходному периоду письмом Банка России от 29.12.2012 г. № 192-т [6] до банков были доведены рекомендации, содержавшие все существенные положения новой методики.

Указанная методика обеспечивает соответствие как Международным стандартам финансовой отчетности, так и документам Базельского комитета по банковскому надзору и может применяться в двух вариантах: базовом и продвинутом. В базовом варианте банк использует только собственные оценки вероятности дефолта, а при продвинутом подходе получает возможность применять также собственные оценки уровня потерь при дефолте, величины кредитного требования, подверженной риску, и срока до погашения требования [1. П. 1.3].

Для перехода на продвинутый вариант методики к внутренней рейтинговой системе банка предъявляются более обширные требования. Параметры, которые банк не имеет возможности оценить при базовом подходе, заменяются установленными табличными значениями, например, ожидаемый уровень потерь для необеспеченных кредитных требований принимается равным $45 \%$ [1. П. 10.8].

Согласно Положению № 483-П внутренняя рейтинговая система банка должна обеспечивать, в частности [1. П. 12.4]:

- раздельную оценку рисков заемщика и рисков финансовых инструментов;

- применение рейтинговой шкалы, построенной на количественных значениях вероятности дефолта заемщиков и содержащей не менее 8 разрядов (градаций);
- отсутствие высокой концентрации в одном разряде шкалы (диапазоны должны быть достаточно узкими).

Для продвинутого варианта методики помимо шкалы заемщиков строится также шкала финансовых инструментов [1. П. 12.6]. Оценки параметров кредитного риска (вероятности дефолта, уровня потерь при дефолте и др.) проводятся по каждому разряду рейтинговой шкалы отдельно. Период наблюдений для оценки вероятности дефолта заемщика должен составлять не менее 5 лет [1. П. 13.8], для оценки уровня потерь по финансовому инструменту - не менее 7 лет по корпоративным заемщикам и не менее 5 лет по розничным заемщикам [1. П. 3.18-3.19]. Внутренние рейтинги банка должны быть включены в процессы управления кредитным риском и принятия решений о выдаче кредита [1. П. 1.6].

Мы полагаем, что одним из значимых последствий перехода банков к применению Положения № 483-п станет отказ от широко распространившихся в начале нашего века эмпирических моделей оценки кредитоспособности и кредитного риска. Внутренние рейтинговые системы банков должны регулярно проходить тестирование статистическими методами [1. П. 12.15]. В сочетании со статистической моделью допускается применять экспертные оценки.

В отличие от банков, все еще использующих Положение № 254-п и вынужденных согласовывать результаты его применения с МСФО (IFRS) 9, порядок создания резервов некредитными финансовыми организациями с 01.01.2017 г. будет полностью соответствовать Международным стандартам финансовой отчетности. С указанной даты вступает в силу Отраслевой стандарт бухгалтерского учета, утвержденный Банком России 01.10.2015 г. № 493-п, содержащий в части признания ссудной задолженности и ее обесценения прямые ссылки на МСФО (IAS) 39 «Финансовые инструменты: признание и оценка» [3. П. 1.6, 1.13 и др.].

Таким образом, российские банки могут использовать (и некоторые из них уже фактически используют) статистические внутренние кредитные рейтинги как инструмент количественного анализа кредитоспособности. Данный инструмент наряду с публичными (общедоступными) кредитными рейтингами является общепринятым в мировой практике.

Публичные кредитные рейтинги компаний эмитентов облигаций составляются как международными, так и национальными рейтинговыми агентствами и широко используются в качестве ориентиров при принятии инвестиционных и финансовых решений. Поэтому для целей настоящего исследования существенное значение имеет вопрос количественной интерпретации рейтинговых категорий. 
Публичные рейтинги присваиваются как отдельным финансовым инструментам (issue credit rating), так и компаниям-эмитентам (issuer credit rating). Кредитный рейтинг эмитента (заемщика) обычно рассматривается как измеритель его кредитоспособности (в частности, такой подход принят и в упомянутом выше Положении № 483-п [3. П. 4.6 и др.]).
В Российской Федерации кредитные рейтинги учитываются не только индивидуальными и институциональными инвесторами, но и органами государственной власти, в частности, при определении надежных активов, в которых должны размещаться собственные средства и страховые резервы страховых организаций $[4,5,8]$ и средства негосударственных пенсионных фондов [7] (табл. 3).

Таблица 3. Ограничения на размещение капитала и резервов страховых организаций и негосударственных пенсионных фондов по кредитному рейтингу эмитента

\begin{tabular}{|l|c|c|}
\hline \multirow{2}{*}{ Рейтинговое агентство } & \multicolumn{2}{|c|}{ Минимальный рейтинг эмитента } \\
\cline { 2 - 3 } & $\begin{array}{c}\text { собственный капитал } \\
\text { и страховые резервы } \\
\text { страховых организаций* }\end{array}$ & $\begin{array}{c}\text { пенсионные резервы } \\
\text { негосударственных } \\
\text { пенсионных фондов ** }\end{array}$ \\
\hline Standard \& Poor's & B-/ ru.BBB & BBB- \\
\hline Fitch Ratings & B-/ BB-(rus) & BBB- \\
\hline Moody's Investors Service & B3 & Baа3 \\
\hline A.M. BestCo & B- & Не используется \\
\hline Эксперт PA & А & Не используется \\
\hline
\end{tabular}

Примечания:

* по международный шкале / по национальной шкале;

** только для облигаций иностранных коммерческих организаций [8. П. 9]

Определения категорий (градаций шкалы) рейтингов обычно даются в терминах вероятности неплатежеспособности (дефолта) или банкротства с использованием шкалы качественных оценок «низкий»- «высокий» (табл. 4).

Таблица 4. Определения укрупненных (базовых) категорий международных рейтингов: Standard \& Poor's (S\&P), Fitch Ratings (Fitch), Moody's Investors Service (Moody's)*

\begin{tabular}{|c|c|c|c|}
\hline \multicolumn{3}{|c|}{ Базовая категория } & \multirow{2}{*}{ Сводное определение категории (сокращенное) } \\
\hline $\mathrm{S} \& \mathrm{P}$ & Fitch & Moody's & \\
\hline A & A & A & $\begin{array}{l}\text { Риск неплатежеспособности практически отсутствует. } \\
\text { Выделяются категории AAA, AA, A (S\&P, Fitch); Aaа, } \\
\text { Aa, A (Moody’s) по степени возрастания риска; для по- } \\
\text { следней категории (А) риск оценивается как «низкий». } \\
\text { Слабая зависимость от отраслевых и макроэкономиче- } \\
\text { ских факторов }\end{array}$ \\
\hline B & B & B & $\begin{array}{l}\text { Умеренный риск, возрастающий от категории ВВВ/Ваa } \\
\text { (S\&P, Fitch / Moody's) к категории В от «низкого» до } \\
\text { «существенного». Значимая зависимость от отраслевых } \\
\text { и макроэкономических факторов }\end{array}$ \\
\hline $\mathrm{C}$ & $\mathrm{C}$ & $\mathrm{Caa}, \mathrm{Ca}$ & $\begin{array}{l}\text { Высокий риск, возрастающий от категории CCC/Caа } \\
\text { (S\&P, Fitch / Moody’s) к категории CC/C/Ca (S\&P / Fitch / } \\
\text { Moody’s) до неминуемого банкротства. Не считая по- } \\
\text { следней категории, наступление или избежание банкрот- } \\
\text { ства определяется отраслевыми и макроэкономическими } \\
\text { факторами }\end{array}$ \\
\hline $\mathrm{R}, \mathrm{SD}, \mathrm{D}$ & $\mathrm{RD}, \mathrm{D}$ & $\mathrm{C}$ & $\begin{array}{l}\text { Банкротство или аналогичные ситуации, такие как } \\
\text { внешнее управление }\end{array}$ \\
\hline
\end{tabular}

* Долгосрочные кредитные рейтинги. Составлено по данным [18, 19, 20].

Международные и национальные рейтинговые агентства не включают в определения категорий точные значения вероятностей, ссылаясь на вариабельность их значений во времени, зависи- мость от отрасли и общего состояния экономики. Тем не менее статистика дефолтов в привязке к кредитным рейтингам регулярно публикуется (табл. 5).

Таблица 5. Статистика корпоративных дефолтов по данным Standard \& Poor's за 1981-2015 гг. (извлечение) [18]

\begin{tabular}{|c|c|c|c|c|c|}
\hline \multirow{2}{*}{ Категория } & \multicolumn{5}{|c|}{ Вероятность банкротства, \%, в течение } \\
\hline & 1 года & 2 лет & 3 лет & 4 лет & 5 лет \\
\hline AAA & 0,00 & 0,03 & 0,13 & 0,24 & 0,35 \\
\hline $\mathrm{AA}$ & 0,02 & 0,06 & 0,13 & 0,23 & 0,34 \\
\hline $\mathrm{A}$ & 0,06 & 0,15 & 0,26 & 0,40 & 0,55 \\
\hline BBB & 0,19 & 0,53 & 0,91 & 1,37 & 1,84 \\
\hline $\mathrm{BB}$ & 0,73 & 2,25 & 4,07 & 5,86 & 7,51 \\
\hline $\mathrm{B}$ & 3,77 & 8,56 & 12,66 & 15,82 & 18,27 \\
\hline $\mathrm{CCC} / \mathrm{C}$ & 26,36 & 35,54 & 40,83 & 44,05 & 46,43 \\
\hline
\end{tabular}

Аналогичные данные доступны в отношении рейтингов Moody's Investors Service в виде «идеализированной кривой кумулятивной вероятности дефолта и величины ожидаемых убытков» [7]. В среднем на горизонте в 5 лет для группы А вероятность неплатежеспособности не превышает 
$0,5 \%$; в группе В наблюдается значительный рост - от 2 \% для категории ВВВ (Ваa) до $20 \%$ и более для категории В; в категориях группы C вероятность банкротства приближается к $50 \%$.

Мы проанализировали связь частоты дефолтов с категориями долгосрочного кредитного рейтинга по данным Standard \& Poor's (рис. 2, по оси ординат - десятичный логарифм вероятности неплатежеспособности). После логарифмирования зависимость для всех рядов оказалась почти строго линейной (все значения $\mathrm{R}^{2}$ в диапазоне $0,97 \ldots 0,99)$.

Данные Moody's Investors Service менее пригодны для такого анализа, поскольку по способу построения являются не фактическими, а сглаженными и интерполированными рядами.

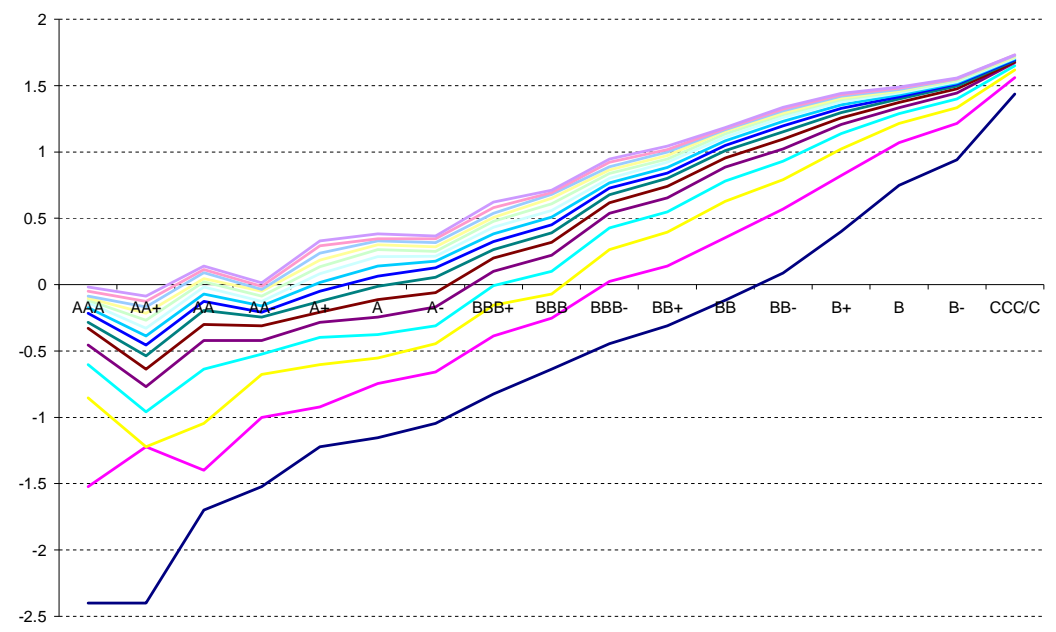

Рис. 2. Зависимость вероятности банкротства от категории долгосрочного кредитного рейтинга (построено по данным Standard \& Poor's за 1981-2015 гг. [18])

Корреляция категории рейтинга Moody's с логарифмом вероятности является достаточно высокой лишь на горизонте от 1 до 4 лет $\left(\mathrm{R}^{2}\right.$ в диапазоне $0,95 \ldots 0,98)$, затем плавно снижается до значений $\mathrm{R}^{2} 0,82 \ldots 0,85$ (на горизонте от 20 до 30 лет). Однако сопоставление категории рейтинга с логарифмом ожидаемого убытка, представленного в том же отчете, дает высокую корреляцию $\left(\mathrm{R}^{2}\right.$ в диапазоне $0,92 . .0,98)$ на всех временных горизонтах, в том числе на 15 -летнем горизонте $\mathrm{R}^{2}$ не ниже 0,95 .

Можно предположить, что полученные результаты отражают принципиальные различия методик построения рейтингов, принятых Standard \& Poor's и Moody's Investors Service, что опровергает допущение о возможности единого подхода к количественной интерпретации категорий кредитных рейтингов. Если подход Standard $\&$ Poor's, дающий логарифмическую зависимость от вероятности дефолта компании, соответствует исходному определению кредитоспособности, то в случаe Moody's рейтинг измеряет скорее средний ожидаемый убыток (вероятность дефолта нелинейно через табличную функцию). Впрочем, на ограниченном временном горизонте (до 4-5 лет) категории рейтинга Moody's относительно надежно сопоставляются и с логарифмом вероятности дефолта.

Необходимо уточнить, что сделанные выше выводы о высокой корреляции относятся лишь к характеру зависимости между категорией рейтинга и вероятностью дефолта, но не к значениям коэффициентов корреляции, которые варьируют- ся по отраслям и в зависимости от стадии экономического цикла. Как было показано Дж. Хильшером и М. Вильсоном, категория долгосрочного рейтинга Standard \& Poor's фактически отражает не столько абсолютную вероятность, сколько «бета-коэффициент вероятности дефолта» («failure beta»), рассчитываемый как коэффициент регрессии по медианной вероятности (табл. 6). В периоды экономического спада возрастает как средняя, так и индивидуальная вероятность дефолта, но значения бета-коэффициентов для конкретных категорий рейтинга остаются стабильными. Таким образом, рейтинг Standard \& Poor's является измерителем относительной вероятности дефолта как пропорции между значением для конкретной категории и медианным средним.

Таким образом, в зависимости от методики построения кредитные рейтинги могут отражать различные количественные аспекты кредитоспособности. Их непосредственное применение в экономическом анализе затрудняется также отсутствием детерминированной связи с показателями финансового анализа. Поскольку методика формирования публичных кредитных рейтингов полностью никогда не раскрывается, а их расчет включает сведения закрытого и эксклюзивного характера, без обращения к рейтинговому агентству невозможно оценить их предстоящее изменение под влиянием экономических решений, например, об увеличении объектом заимствования. Примером закрытых данных в составе информационной базы публичных рейтингов явля- 
ются кредитные истории (даже если соответствующая информация в отношении отдельной компании доступна аналитику по запросу, для построения рейтинга необходимо иметь такую информацию по всем входящим в рейтинг компаниям). Эксклюзивные сведения составляют, в частности, экспертные оценки, сделанные по заказу рейтингового агентства. Поэтому публичные кредитные рейтинги хорошо подходят для принятия решений об инвестициях в корпоративные облигации, но не для решений о кредитовании.

Таблица 6. Зависимость бета-коэффициентов вероятности дефолта от категории кредитного рейтинга Standard \& Poor's [23]

\begin{tabular}{|c|c|}
\hline Рейтинг S\&P & $\begin{array}{c}\text { Бета-коэффициент вероятности } \\
\text { дефолта }\end{array}$ \\
\hline $\mathrm{AAA}$ & 0,38 \\
\hline $\mathrm{AA}+$ & 0,52 \\
\hline $\mathrm{AA}$ & 0,53 \\
\hline $\mathrm{AA}-$ & 0,57 \\
\hline $\mathrm{A}+$ & 0,60 \\
\hline $\mathrm{A}$ & 0,61 \\
\hline $\mathrm{A}-$ & 0,73 \\
\hline $\mathrm{BBB}+$ & 0,86 \\
\hline $\mathrm{BBB}$ & 0,91 \\
\hline $\mathrm{BBB}-$ & 1,10 \\
\hline $\mathrm{BB}+$ & 1,78 \\
\hline $\mathrm{BB}$ & 2,10 \\
\hline $\mathrm{BB}-$ & 2,57 \\
\hline $\mathrm{B}+$ & 3,36 \\
\hline $\mathrm{B}$ & 4,60 \\
\hline $\mathrm{B}-$ & 4,91 \\
\hline $\mathrm{CCC}+$ & 4,96 \\
\hline $\mathrm{CCC}$ & 8,44 \\
\hline
\end{tabular}

Внутренние кредитные рейтинги банков, в отличие от публичных, вполне подходят для принятия кредитных решений, так как при необходимости могут быть пересчитаны для конкретного заемщика в зависимости от сценариев предполагаемого кредитования. Однако такой способ недоступен для внешних по отношению к банку аналитиков, в том числе для самих заемщиков. Кроме того, внутренние рейтинги, присвоенные одному и тому же экономическому субъекту различными банками, могут существенно различаться. Таким образом, внутренние рейтинги представляют собой оценку кредитоспособности с позиции конкретного субъекта анализа - банка, учитывающую сложившиеся и ожидаемые условия его деятельности, стратегические интересы и текущие предпочтения.

Возможность множественных оценок кредитоспособности одного и того же экономического субъекта не отменяет объективного характера самого данного понятия, поскольку кредитоспособность принято рассматривать в привязке не только к заемщику, но и к кредитному договору [10]. Однако рассмотрим ситуацию, когда заемщик обратился в два различных банка для выяснения потенциальной возможности и условий выдачи кредита на фиксированную сумму и под заранее определенное обеспечение. Если исходить из того, что возможность полностью и в срок рассчитаться по обязательствам не зависит от того, в каком из двух банков будет открыта кредитная линия, кредитоспособность не может быть субъективным понятием. При этом оценки кредитоспособности, полученные банками, могут различаться в зависимости от примененных моделей и доступной информационной базы, но апостериорная статистика частоты дефолтов будет независимым измерителем. Поэтому как минимум количественная интерпретация кредитоспособности должна оставаться единой для всех субъектов экономического анализа.

В связи с проблемами закрытости информации о методиках и информационной базе публичных и внутренних кредитных рейтингов представляют интерес общедоступные методики, индикаторы и показатели, представленные в научных работах зарубежных и отечественных авторов. Их значимыми достоинствами являются также верифицируемость и постоянное совершенствование в процессе научных дискуссий. Хотя информационная база расчета соответствующих показателей обычно менее обширна, чем у рейтинговых агентств и крупных коммерческих банков, необходимые для проверки сведения могут быть получены, а расчеты проведены при сравнительно незначительных затратах. Кроме того, общепринятым методом доказательства надежности новых моделей оценки кредитоспособности является сравнение их предсказаний с данными международных и национальных рейтинговых агентств. Как правило, такие модели показывают сопоставимые или даже превосходящие результаты. Недостатком для практического применения является другой аспект: отсутствие поддержки модели. За редкими исключениями авторы не склонны на протяжении многих лет адаптировать модель к изменяющимся условиям бизнес-среды и расширяющимся возможностям информационной базы, а также повышать надежность прогнозов. Новые разработки тех же или других авторов могут настолько отличаться от предшествующих, что для экономических субъектов, решивших их использовать, затраты на изменение сложившейся организации анализа станут существенными. Также остается проблема выбора оптимальной методики из спектра существующих: как правило, нет варианта с бесспорными преимуществами по всем значимым параметрам. В перечне значимых параметров выбора методики оказываются, как минимум, ее универсальность (в отношении размеров компаний, отраслей, регионов, ожидаемой длительности применения без потери качества прогнозов), надежность и издержки применения (явные - трудовые и финансовые - и вмененные, такие как выигрыш от оперативности построения прогнозов). К числу проблем выбора можно отнести и вероятное отсутствие пригодной и доступной некоммерческой модели для нужного региона или сектора экономики. 
За последние десятилетия к решению задачи оценки кредитоспособности как уровня кредитного риска или вероятности дефолта применялись десятки различных подходов. Ограничиваясь лишь теми из них, что дают экономически содержательные количественные оценки, мы должны в первую очередь отметить популярные модели логистической регрессии (логит-модели).

Результативным показателем логит-моделей является вероятность того или иного события, рассчитанная (аппроксимированная) при помощи логит-функции:

$$
P=\frac{1}{1+e^{-Z}},
$$

где $P$ - вероятность события, коэффициент в диапазоне $[0 ; 1] ; Z$ - результат вычисления линейной функции следующего вида:

$$
Z=a_{0}+a_{1} X_{1}+a_{2} X_{2}+\ldots+a_{m} X_{m},
$$

где $a_{0}, a_{1}, \ldots, a_{m}$ - числовые коэффициенты (коэффициенты логит-регрессии); $X_{0}, X_{1}, \ldots, X_{m}-$ факторные переменные (независимые переменные логит-регрессии).

Значение $Z=0$ соответствует вероятности $P=0,5$ (50\%), значения $Z<0$ - вероятности менее $50 \%, Z>0$ - вероятности свыше $50 \%$. Зависимая переменная $Y$ для логит-регрессии в простейшем случае принимает два возможных значения: 0 или 1.

Популярность применения логит-регрессии для прогнозирования банкротств связана с достаточно высокой надежностью получаемых результатов при более слабых ограничениях на входные данные по сравнению с классическим линейным или квадратичным дискриминантным анализом. Применительно к анализу кредитоспособности логит-модели можно применить непосредственно оценки вероятности или для классификации компаний по вероятности дефолта. Минимальная шкала классификации обычно включает 3 группы: низкую вероятность дефолта (например, ниже $5 \%$ ), высокую вероятность дефолта (соответственно выше $95 \%$ ) и зону неопределенности между ними (интервал от 5 до $95 \%$ ).

Таким образом, если в рассмотренных выше кредитных рейтингах возможны вариации при количественной интерпретации категорий, то логит-модели дают оценку строго в терминах), что означает:

- произвольный («эмпирический») выбор исходных показателей;

- как правило, произвольный переход от фактических значений показателей к категориям по бинарной или ступенчатой шкале - вероятности дефолта. Для учета размера ожидаемых потерь и прочих факторов кредитного риска необходимо вводить дополнительные функции (фактически использовать дополнительную модель).

Необходимо также отметить, что значительный прогресс, достигнутый за последние 20 лет в области прогнозирования корпоративных дефолтов, был связан не столько с применением самих логит-моделей, сколько с включением в них макроэкономических и отраслевых переменных. Наиболее известным из первых удачных результатов в данной области является исследование T. Вильсона [24], повлиявшее на многие последующие работы. Типичными макроэкономическими переменными, влияющими на частоту дефолтов, являются темпы роста валового внутреннего продукта (а также их изменение по сравнению с предыдущим годом, позволяющее учесть стадию экономического цикла), уровень процентных ставок, доходность фондовых индексов (например, индекса Standard \& Poor's);

- учитывая, что средняя (медианная) частота корпоративных дефолтов действительно в значительной степени зависит от макроэкономических переменных, а индивидуальный риск компании может быть учтен при помощи введения в модель соответствующих финансовых показателей, комплексный подход Вильсона представляется весьма перспективным для построения внутренних кредитных рейтингов коммерческих банков. К сожалению, последние доступные обзоры банковской практики (например, $[14,15])$ показывают, что большинство внутренних рейтингов строится на моделях с эмпирическим агрегированием дискретизированных и экспертных оценок (далее также «эмпирические» модели являются дискретизацией с низким разрешением);

- произвольный подход к обобщению оценок финансовых и производственных показателей (с «эмпирическими» весовыми коэффициентами) и к их слиянию с качественными экспертными оценками.

Вышесказанное не означает, что существующие внутренние рейтинги российских банков непригодны для использования, поскольку в систему управления кредитными рисками встроены процедуры их верификации и корректировки, a «произвольный выбор» исходных показателей обычно экономически обоснован. Общепринятая в российской практике процедура дискретизации также имеет практический смысл в отсутствие информационной базы для построения надежных статистических моделей и при наличии значимых сомнений в достоверности бухгалтерской отчетности (не столько из-за преднамеренных искажений, сколько из-за несовершенства отечественной методологии учета). Принципиальные недостатки подобных моделей состоят в следующем:

- эмпирический подход к построению модели кредитного риска не обеспечивает ее оптимальности. Статистическая модель на том же наборе исходных данных и экспертных допущений, которые были задолжены в эмпирическую модель, позволяет достичь наилучшей возможной надежности прогноза. Определенные трудности состоят 
лишь в формализации экспертных допущений и прогнозов, компенсирующих неполноту исходных данных и ожидаемые отклонения от исторических тенденций и соотношений;

- трудно дать априорную количественную оценку надежности эмпирической модели, т.е. рассчитать ожидаемый процент ошибок первого и второго рода. Метод тестирования на контрольной выборке применим и к эмпирической модели как к «черному ящику», но для достижения удовлетворительных результатов объем данных должен быть достаточно большим, тогда как «эмпирический подход» обычно выбирается именно изза недостаточности или нерепрезентативности выборки;

- итоговая оценка, полученная по эмпирической модели, является условной и не может быть соотнесена с измеримыми экономическими показателями. Кроме того, такая оценка непригодна к использованию в качестве входящей переменной для решения иных задач.

Последнее обстоятельство не позволяет с учетом ранее сформулированных требований использовать рейтинги заемщиков, присвоенные им на основе эмпирических моделей, в качестве количественной оценки кредитоспособности. Применительно к упомянутым достоинствам современных логит-моделей риска дефолта стоит также отметить, что по данным одного из наиболее полных обзоров российской банковской практики только один банк из пяти явным образом учитывал макроэкономические факторы. Таким образом, на текущий момент широкое внедрение современных статистических моделей кредитного риска остается актуальной задачей для российских кредитных организаций.

Среди статистических методов анализа, дающих пригодные для использования количественные оценки, необходимо также назвать линейный дискриминантный анализ, на основе которого были построены исторически первые модели прогнозирования банкротств. Несмотря на давнее смещение интереса экономистов в пользу более эффективных логит-моделей, дискриминантный анализ в конкретных ситуациях может давать столь же и даже более надежные результаты [21]. Результативный показатель линейных дискриминантных моделей представляет собой безразмерный коэффициент (Z-score), но каждое его значение однозначно соотносится с уровнем вероятности. Таким образом, с точки зрения интерпретации количественной оценки данные модели ничем не отличаются от логистических (за исключением необходимости одного дополнительного преобразования).

В качестве одного из наиболее интересных результатов из числа полученных за последние годы методом линейного дискриминантного анализа мы хотели бы отметить «пересмотренную модель Альтмана для развивающихся рынков», обычно обозначаемую как «Z”-model» или «Z"-score». В современном виде модель была опубликована Э. Альтманом и Э. Хотчкиссом в 2006 г. [12] и обладает следующими преимуществами:

- применима к экономическим субъектам любой отрасли как производственного, так и непроизводственного сектора;

- распространяется на компании, не являющиеся эмитентами ценных бумаг, но пригодна и по отношению к эмитентам;

- адаптирована к развивающимся рынкам, хотя пригодна и для стран с развитой рыночной экономикой. $\mathrm{K}$ числу стран с развивающимися рынками традиционно относят страны BRICS (Бразилия, Россия, Индия, Китай, ЮАР), Восточной Европы, Мексику и др.

Изначально модель калибровалась на примере Мексики, но затем ее работоспособность была подтверждена тестированием на данных Италии [21], Китая [25] и др. Тот факт, что Z” прошла все тесты практически без потерь надежности, подтверждает ее высокую устойчивость к региональному фактору. Текущая версия модели Z" имеет вид

$$
Z^{\prime \prime}=3,25+6,56 \frac{W C}{T A}+3,26 \frac{R E}{T A}+6,72 \frac{E B I T}{T A}+1,05 \frac{B E}{T L},
$$

где $Z$ ” - значение Z"-score, коэф.; $W C$ - чистый оборотный капитал как разность между оборотными активами и краткосрочными обязательствами; $T A$ - совокупные активы; $R E$ - нераспределенная прибыль по балансу; $E B I T$ - прибыль до уплаты процентов и налогов; $B E$ - чистые активы по балансу; $T L$ - совокупные обязательства по балансу.

Значение $Z$ ” = 0 соответствует уровню дефолта. В рамках настоящей работы особенно интересно установленное авторами соответствие между значениями Z и категориями кредитного рейтинга Standard \& Poor's (табл. 7). За счет этого расширения Z" стала более гибкой (увеличилось количество классификационных групп; для предыдущей модификации использовалось стандартное деление на три группы), наглядной и пригодной для непосредственного определения кредитного рейтинга. Однозначное соответствие между значением Z-score, кредитным рейтингом по шкале S\&P и уровнем вероятности дефолта представляет ценность само по себе, но на сегодняшний день это еще и единственная общедоступная модель из представленных зарубежными авторами, потенциально пригодная для применения в России. Достаточно успешные попытки определять категории S\&P статистическими методами многократно предпринимались как ранее, так и впоследствии, но большинство из них ограничено компаниями-эмитентами облигаций и практически все построены на примере Соединенных Штатов. 
Таблица 7. Соответствие между категориями кредитного рейтинга Standard \& Poor's (S\&P) и значениями Z"-score [12]

\begin{tabular}{|c|c|c|}
\hline Рейтинг S\&P & $\begin{array}{c}Z \text { "для нижней } \\
\text { границы группы }\end{array}$ & Риск дефолта \\
\hline AAA & 8,15 & \multirow{10}{*}{ Безопасная зона } \\
\hline $\mathrm{AA}+$ & 7,60 & \\
\hline AA & 7,30 & \\
\hline AA- & 7,00 & \\
\hline $\mathrm{A}+$ & 6,85 & \\
\hline $\mathrm{A}$ & 6,65 & \\
\hline A- & 6,40 & \\
\hline BBB+ & 6,25 & \\
\hline BBB & 5,85 & \\
\hline BBB- & 5,65 & \\
\hline $\mathrm{BB}+$ & 5,25 & \multirow{5}{*}{ «Серая зона» } \\
\hline BB & 4,95 & \\
\hline BB- & 4,75 & \\
\hline $\mathrm{B}+$ & 4,50 & \\
\hline B & 4,15 & \\
\hline B- & 3,75 & \multirow{5}{*}{ Зона потерь } \\
\hline CCC+ & 3,20 & \\
\hline CCC & 2,50 & \\
\hline CCC- & 1,75 & \\
\hline D & 0,00 & \\
\hline
\end{tabular}

Мы полагаем, что объединяя оценки Z" с данными о частоте дефолтов из годовых отчетов Standard \& Poor's и учитывая отраслевые и макроэкономические факторы для корректировки медианного среднего, можно получить достаточно надежную, верифицируемую и дешевую в использовании модель риска дефолта.

В отношении практического применения такой модели к оценке кредитоспособности необходимо сделать два существенных замечания. Вопервых, несмотря на разницу понятий «нарушение обязательств по кредитному договору» (из определения кредитоспособности) и «дефолт по обязательствам» (событие, которое прогнозируют рассмотренные выше модели), особенности определения дефолта зарубежными ученымиэкономистами и рейтинговыми агентствами позволяют считать соответствующие модели вполне пригодными с этой точки зрения. Рейтинговые агентства Standard \& Poor's, Fitch Ratings и Moody's классифицируют как дефолт в том числе задержку платежей на определенный срок (в общем случае 30 дней), а в качестве обязательств принимают задолженность по облигациям и иные заимствования, по которым платятся проценты. Информация по текущей, в том числе торговой кредиторской задолженности, рассматривается в последнюю очередь, когда задержка платежей по ней становится причиной иска о банкротстве или другим способом попадает в базы данных бюро кредитных историй или самих рейтинговых агентств. При столь серьезных проблемах организации вопрос о регулярном исполнении обязательств по кредитам уже не возникает.

Во-вторых, для оценки кредитоспособности помимо вероятности дефолта важно знать уровень ожидаемых потерь, который общедоступные модели обычно не прогнозируют. Как показал ряд исследований, уровень потерь неправомерно счи- тать ни константой, ни независимой по отношению К вероятности дефолта переменной. В частности, критика многими зарубежными экономистами ранних вариантов Базельской системы управления кредитными рисками основывалась именно на невнимании к явной обратной зависимости риска дефолта и уровня ожидаемых потерь [13]. Последний вариант документов «Базель II» (отраженный в том числе в Положении № 483-п) был откорректирован с учетом данной критики, причем банкам было рекомендовано обращать внимание на такую зависимость, но оценивать ее наличие или отсутствие самостоятельно. Поэтому отсутствие надежной модели уровня потерь может стать значимым препятствием в применении общедоступных моделей риска дефолта. Кроме того, отметим, что внедрение любой внешней разработки во внутреннюю банковскую практику может проводиться лишь при подтверждении ее полного соответствия требованиям Банка России, надлежащем предварительном тестировании и последующей систематической верификации. Даже если общедоступная модель успешно пройдет этап внедрения (для чего ее точность может оказаться недостаточной), на последующих этапах верификации неизбежная корректировка может изменить ее до степени полной несхожести с оригиналом. При этом модель неизбежно попадет под ограничения на распространение информации, содержащей коммерческую тайну, и потеряет преимущества общедоступной публично верифицируемой методики. Для всех прочих субъектов анализа, кроме банков, следование стратегии внедрения общедоступных разработок не связано со столь значительными проблемами.

Противопоставляя модели вероятности дефолта рейтингам банков и международных агентств, необходимо отметить, что те же агентства и крупные консалтинговые компании также активно разрабатывают и поддерживают модели количественного прогнозирования банкротства (например, закрытая модель RiskCalc от агентства Moody's [22]).

Помимо более обширной информационной базы, более сложных и трудоемких аналитических процедур, непрерывной верификации и постоянного совершенствования модели, пользователям предоставляется ряд дополнительных возможностей. Так, перестает быть проблемой учет размера ожидаемых потерь, качества обеспечения, гарантий и прочих факторов кредитоспособности, равно как и трансформация рейтинга в вероятность. Частично компенсируется и проблема пересчета: поскольку модель предоставляется клиентам не как методика, а как сервис, можно в реальном времени оценить последствия финансовых решений. Также не возникает вопрос измерителя: заказчик получает оценку в удобном ему формате (из списка возможных). 
Некоторые затруднения могут возникнуть только при изменении методики самим консалтинговым агентством, когда клиентам необходимо будет нивелировать соответствующие изменения рейтингов. Возможность эффективного использования указанных сервисов в российской банковской практике, в том числе с позиции требований Положения № 483-п, должна быть предметом отдельного исследования.

Рассмотрев наиболее распространенные подходы к анализу кредитоспособности с точки зрения формирования универсального количественного показателя, мы можем сделать следующие выводы:

- оценка кредитоспособности при помощи публичных и внутренних кредитных рейтингов, общедоступных моделей дефолта и коммерческих моделей кредитного риска позволяет получить несколько количественных показателей, отвечающих требованиям экономического содержания и полезности для принятия решений, в том числе вероятность дефолта $(P D)$ и уровень потерь при дефолте $(L G D)$;

- показатели «вероятность дефолта» и «уровень потерь при дефолте» имеют существенное значение для оценки кредитоспособности, но по отдельности не могут считаться релевантными и комплексными характеристиками.

Исходя из выбранного подхода и сформулированных в начале настоящего подраздела требований, мы считаем, что лучшим количественным показателем кредитоспособности будет средневзвешенный ожидаемый уровень потерь $(E L)$ :

$$
E L=P D \times L G D,
$$

где $E L$ - средневзвешенный ожидаемый уровень потерь, коэф.; $P D$ - вероятность дефолта, коэф.; $L G D$ ожидаемый уровень потерь при дефолте, коэф.

В формуле (4) мы считаем $L G D$ рассчитанным по аналогии с нормированным ожидаемым убытком, т.е. учитывающим все возмещения, издержки их получения и распределение денежных потоков во времени. Для оценки кредитоспособности заемщика $E L$ следует рассчитывать в отношении всех обязательств, по которым экономических субъект уплачивает проценты (т.е. за исключением, например, текущей кредиторской задолженности). При перспективной (прогнозной) оценке кредитоспособности в сумму таких обязательств будут включены ожидаемые к получению кредиты (займы), выпуски облигаций и т.д. Ожидаемый уровень потерь по конкретному обязательству (например, по кредитному договору) может отличаться от количественной оценки кредитоспособности заемщика из-за условий предоставления кредита (займа), действующих законодательных требований (таких как очередность погашения обязательств при недостаточности денежных средств), различия в сроках погашения обязательств и графиках выплат.
Чтобы оценка кредитоспособности была достоверной, сумма обязательств, подверженных риску дефолта $(E A D)$, по отношению к которой рассчитывается уровень потерь $(L G D)$, должна определяться методом дисконтированных денежных потоков по первоначальной эффективной процентной ставке (поскольку таким способом оцениваются ожидаемые потери). Если кредит выдается одной суммой, $E A D$ будет совпадать с ней на момент принятия решения и на момент выдачи. В последующих периодах $E A D$ будет уменьшаться по мере погашения обязательств. Таким образом, показатели $P D, L G D$ и $E A D$ будут соответствовать требованиям МСФО (IAS) 9 «Финансовые инструменты». Для банков как субъектов анализа кредитоспособности $P D, L G D$ и $E A D$, рассчитанные по методике Постановления № 483-П, будут наилучшими оценками. Для прочих субъектов анализа кредитоспособности данные показатели могут быть получены:

- из открытых источников, таких как отчеты рейтинговых агентств $(P D, L G D)$ и публикуемая финансовая отчетность ( $E A D$ как сумма кредитных и аналогичных им обязательств, $P D$ и $L G D$ как функции моделей дефолта). Такой подход является единственно возможным при внешнем анализе. Если рейтинговое агентство или консалтинговая компания предоставляют клиентам доступ к собственным оценкам $E L$, в отношении конкретных компаний, необходимость в самостоятельном расчете через $P D$ и $L G D$ отсутствует;

- путем моделирования денежных потоков и анализа сценариев развития бизнеса. Такой подход возможен при внутреннем анализе. В любом случае целесообразно сравнить полученные значения $P D$ и $L G D$ с внешними оценками, полученными от рейтинговых агентств или путем применения к данным финансовой отчетности одной из моделей прогнозирования дефолта. Внутренний анализ позволяет точнее учесть индивидуальные особенности экономического субъекта, но использует большое количество экспертных оценок и допущений, вследствие чего иногда может давать нереалистичные результаты.

На наш взгляд, задачи внутреннего анализа кредитоспособности должны состоять не столько в ее точной оценке (поскольку банки принимают кредитные решения на основании своих собственных оценок, воспроизвести которые без доступа к их информационной базе затруднительно), сколько в выявлении факторов изменения. Определив причины роста или снижения кредитоспособности и прогнозируя ее изменения в зависимости от разрабатываемых инвестиционных и финансовых решений, руководство экономического субъекта обеспечивает эффективное управление рисками и капиталом. Поэтому возможный подход к внутреннему анализу состоит в оценке влияния исторических изменений и будущих ре- 
шений на ключевые показатели финансовой отчетности, чтобы затем применить к ним статистические модели, релевантные для субъектов внешнего анализа.

Использование количественной оценки кредитоспособности в принятии решений должно производиться с учетом зависимости «доходность - риск», где доходность по конкретному обязательству измеряется эффективной процентной ставкой. Применительно к шкале кредитных рейтингов Moody's необходимые ориентиры уже содержатся в таблицах «идеализированной кривой дефолтов и потерь» [24], тогда как шкалу рейтингов $\mathrm{S} \& \mathrm{P}$, построенную по $P D$, необходимо корректировать с учетом $L G D$. При этом для организаций-кредиторов целесообразно устанавливать не единственное значение порога отсечения по $E L$, а ограничения на удельный вес обязательств в разбивке по уровням кредитного риска и с учетом иных факторов (как минимум, экономической природы - кредиты, облигации, гарантии - и сроков погашения). На наш взгляд, значимым преимуществом подхода к управлению рисками по средневзвешенным ожидаемым убыткам $E L$ в сравнении с классификацией по консервативным оценкам (среднее + надбавка) являются избежание двойного счета риска и однозначное выявление отклонений (выхода за установленные пределы риска по группе обязательств).

В заключение отметим, что однозначная количественная интерпретация кредитоспособности имеет большое значение, поскольку позволяет надежно оценивать существующие и разрабатываемые методики анализа с позиции релевантности и практической значимости результативных показателей. Мы надеемся, что сформулированные нами требования и обоснованный подход к количественному измерению кредитоспособности будет полезен специалистам-практикам при выборе из множества доступных моделей и методик.

\section{Литература}

1. Положение о порядке расчета величины кредитного риска на основе внутренних рейтингов: утв. Банком России 06.08.2015 №483-п // СПС «КонсультантПлюс».

2. Положение о порядке формирования кредитными организациями резервов на возможные потери по ссудам, по ссудной и приравненной к ней задолженности: утв. Банком России 26.03.2004 №254-п // СПС «КонсультантПлюс».

3. Положение «Отраслевой стандарт бухгалтерского учета некредитными финансовыми организациями операций по выдаче (размещению) денежных средств по договорам займа и договорам банковского вклада»: утв. Банком России 01.10.2015 №493-п // СПС «КонсультантПлюс».

4. О порядке инвестирования собственных средств (капитала) страховщика и перечне разрешенных для инвестирования активов: указание Банка России от 16.11.2014 №3445-у // СПС «КонсультантПлюс».

5. О порядке инвестирования средств страховых резервов и перечне разрешенных для инвестирования активов: указание Банка России от 16.11.2014 №3444-у // СПС «КонсультантПлюс».

6. О Методических рекомендациях по реализации подхода к расчету кредитного риска на основе внутренних рейтингов банков: письмо Банка России от 29.12.2012 №192-т // СПС «КонсультантПлюс».
7. Об утверждении Правил размещения средств пенсионных резервов негосударственных пенсионных фондов и контроля за их размещением: Постановление Правительства РФ от 01.02.2007 №63 // СПС «КонсультантПлюс».

8. Перечень рейтинговых агентств, утвержденный Советом директоров Банка России 26.12.2014 // Банк России: офиц. сайт. URL: http://cbr.ru/press/pr.aspx?file=26122014_ 180242if2014-1226t17_58_58.htm.

9. Международный стандарт финансовой отчетности (IFRS) 9 «Финансовые инструменты»: введ. приказом Минфина России от 27.06.2016 №98н // СПС «КонсультантПлюс».

10. Ендовицкий Д.А. Анализ и оценка кредитоспособности заемщика: учеб.-практ. пособие / Д.А. Ендовицкий, И.В. Бочарова. М.: КНОРУС, 2008. 263 c.

11. Финансовый менеджмент: учеб. / Д.А. Ендовицкий, Н.Ф. Щербакова, А.Н. Исаенко и др.; под общ. ред. Д.А. Ендовицкого. 2-е изд., испр. и доп. М.: Рид Групп, $2012.800 \mathrm{c}$.

12. Altman E.I. Corporate Financial Distress and Bankruptcy / E.I. Altman, E. Hotchkiss. 3rd ed. New York: John Wiley and Sons, 2006. 368 p.

13. Altman E.I. Credit Ratings and the BIS Reform Agenda (2001) / E.I. Altman, A. Saunders // Stern School of Business, New ork: official site. URL: http://pages.stern.nyu. edu/ ealtman/ CREDIT_RATINGS_AND_THE_BIS_REFORM_AGENDA1.pdf

14. Сквориова Н.К. Анализ методик оценки кредитоспособности юридических лиц / Н.К. Скворцова, Л.А. Проскурякова, И.Н. Зенкин // Управление экономическими системами. 2013. № 6. URL: http://uecs.ru/uecs54-542013/item/2224-2013-06-28-10-53-00.

15. Финогеев Д.Г. Оценка кредитоспособности юридических лиц на примере крупнейших банков Российской Федерации / Д.Г. Финогеев, Е.М. Щербаков // Современные проблемы науки и образования. 2013. № 6. URL: http://www.scienceeducation.ru/ru/article/view?id=10779.

16. A glossary of terms used in payments and settlement systems // Bank for International Settlements: Committee on Payment and Settlement Systems: офиц. сайт. URL: http://www. bis.org/cpmi/glossary_030301.pdf

17. Definitions of Ratings and Other Forms of Opinion // Fitch Ratings: official site. URL: http://www.fitchratings.com/ web_content/r atings/fitch_ratings_definitions_and_scales.pdf

18. S\&P Global Ratings Definitions // Standard \& Poor's: official site. URL: http://www.standardandpoors.com/en_EU/web/ guest/article/-/view/type/PDF/sourceAssetId/1245208814148.

19. Rating Symbols and Definitions // Moody's Standing Committee on Rating Symbols and Definitions. Moody's Investors Service: official site. URL: http://www.moodys.com/sites/products/ AboutMoodysRatingsAttachments/MoodysRatingSymbolsandDefinitions.pdf

20. 2015 Annual Global Corporate Default Study And Rating Transitions // Standard \& Poor's Ratings Services. Standard \& Poor's: official site. URL: http://www.spratings.com/documents/ 20184/ 774196/2015 Annual Global Corporate Default Study And Rating Transitions/6d311074-5d56-4589-9ef8-a43615a6493b.

21. Altman E.I. Predicting Financial Distress of Companies: Revisiting the Z-Score and Zeta(R) Models (7/2000) / E.I. Altman // Stern School of Business, New York: official site. URL: http:// pages.stern.nyu.edu/ ealtman/Zscores.pdf

22. Falkensten E. "RiskCalc ${ }^{\mathrm{TM}}$ Private Model: Moody's Default Model for Private Firms" // E. Falkensten, A. Boral, L. Carty // Global Credit Research. 2000. № 5 (May).

23. Hilscher J. Credit ratings and credit risk / J. Hilscher, M. Wilson // International Business School, Brandeis University; Saïd Business School, Oxford University. URL: http://www. brandeis. edu/departments/economics/RePEc/brd/doc/Brandeis_WP31.pdf

24. Moody's 30-year Idealised Cumulative Expected Default and Loss Rates // Moody's Investors Service: official site. URL: http://www.moodys.com/researchdocumentcontentpage.aspx?docid=P BS_SF434522.

25. Zhang L. Corporate financial distress diagnosis model and application in credit rating for listing firms in China / L. Zhang, E.I. Altman, J. Yen // Frontiers of Computer Science. 2010. № 4(2). P. 220-236. 